\title{
Why has the rise in obesity not reversed the decline in cardiovascular mortality? Cardiometabolic death rate trends in Brazil (1980-2011)
}

\section{Por que o aumento da obesidade não reverte o declínio da mortalidade por doença cardiovascular? Tendências de mortalidade por evento cardiometabólico no Brasil (1980-2011)}

Paulo Andrade Lotufo'

Faculdade de Medicina da Universidade de São Paulo (FMUSP), São Paulo, Brazil

'MD, PhD. Titular Professor, Discipline of Internal Medicine, Faculdade de Medicina da Universidade de São Paulo (FMUSP), São Paulo, Brazil.
In the November 2000 issue of this journal, the editorial made a prediction that the decreasing trend of cardiovascular death rates in Brazil that had been seen in the 1990s would flatten out, as the first effect of the increasing prevalence of obesity in this country. ${ }^{1}$ The premises for this statement were consistent: as the average body mass index increases, diabetes prevalence rates consequently rise and act directly on the incidence and case-fatality rates of cardiovascular diseases, thus causing the descending trend of cardiovascular deaths to flatten out and then possibly start an upward trend. Fourteen years after this editorial, I regret to inform all our readers that this prophecy (which I wrote) did not come to pass. The reality is that obesity is increasing in Brazil, ${ }^{2}$ as it is everywhere, ${ }^{3}$ and cardiovascular mortality rates are declining in Brazil, ${ }^{4}$ as in most other countries. ${ }^{5}$

To explain this apparent paradox, hypotheses such as the notion that the body mass index is not a good surrogate of adiposity as a risk for atherosclerosis and high blood pressure have been put forward. ${ }^{6}$ Another reason may relate to the improvement of medical care, starting with greater control over risk factors at the primary care level, and especially the introduction of new protocols for cardiovascular emergency and a role for statins/aspirin/beta-blockers after myocardial infarction. ${ }^{7}$ Another possibility is that this paradox may have been caused by a nosological artifact: a shift in the underlying cause of death from cardiovascular diseases to diabetes.

The rationale for considering that there may be a classification bias is that, in most cases, people with diabetes die due to heart disease and stroke. However, this has been changing since 1996, with the adoption of the $10^{\text {th }}$ revision of the International Classification of Diseases (ICD-10), in which both diabetes and a well-defined final cardiovascular event can be considered to be the underlying cause of death. This nosological artifact has been found not to be limited to Brazil and has also been shown in countries like Mexico and the United States. ${ }^{8}$ This rationale has been used recently for comparisons of mortality data between countries. ${ }^{9}$

To test this hypothesis, I analyzed the trends in assignment of the underlying cause of death over a period of three decades from 1980 to 2011, according to gender. This analysis concentrated on the age group from 30 to 69 years, because there are few cardiovascular deaths at younger ages (only 2.5\% of cardiovascular deaths were among individuals under 30 years of age during this period), and because a high proportion of such deaths among individuals over the age of 70 years cannot be compared due to comorbidities. Since Brazil adopted ICD-10 only in 1996, mortality data were classified according to the $9^{\text {th }}$ revision (ICD-9) from 1980 to 1995 and thereafter using ICD-10. I analyzed all cardiovascular diseases (chapters VII and IX of ICD-9 and ICD-10, respectively), coronary heart disease (CHD, codes: 410-5 and I-20-25) and stroke (code: 460-8 and I-6169), separately. Furthermore, I added the cases with diabetes (codes: 205 and E-10-15) to all the cardiovascular diseases, thus creating a category called "cardiometabolic deaths." 
The investigation plan included: (1) review of the numbers in all the categories above, with calculation of the proportion of deaths relating to diagnoses that were listed as ill-defined causes of death; (2) adjustment of all crude death rates using the World Standard Population; (3) calculation of temporal changes to death rates using the Joinpoint 4.1 .1 software. ${ }^{10}$

Figure 1 displays the temporal trends of death rates due to cardiovascular diseases and diabetes for men and women in Brazil from 1980 to 2011. The average percentage change for men was different between the periods 1980-94 and 1994-2011, with a faster pace in the second period. For women, a decline in rates was observed from 1980 to 1992; the rates flattened out in 1992-96; and a new downward trend began in 1996. Despite the improvement in coverage of the mortality information system and more reliable quality of death notification, which could have artificially inflated the rates, a persistent decline for cardiometabolic death rates can be seen in all periods. By examining a period with good quality of mortality data, such as 2005 to 2011, it was possible to measure the average annual percentage change in cardiovascular diseases, CHD, stroke and diabetes (Table 1). Significant declines in rates were estimated for all these diseases, except for diabetes in men. Comparison between the sexes showed that only for $\mathrm{CHD}$ was the reduction in rates faster for women.
These temporal changes to death rates in Brazil show that the decline is occurring in the whole country, despite the shifting of nosological causes from cardiovascular diseases to diabetes. The faster decline of stroke death rates, compared with CHD, reveals that the phase of "delayed cardiovascular epidemiological transition" has ended. ${ }^{11}$ Moreover, the agenda for cardiovascular epidemiology needs to deeply consider the meaning of adiposity as a risk factor. Unfortunately, the early studies by Jean Vague in the 1950s, ${ }^{11,12}$ in which the components of obesity were evaluated separately, were endorsed by

Table 1. Annual average percentage change (and 95\% confidence interval) of age-adjusted death rates in Brazil over the period 2005 to 2011

\begin{tabular}{lcc} 
& Men & Women \\
All cardiovascular diseases & -2.12 & -2.34 \\
(ICD-10: chapter IX) & $(-2.41$ to -1.82$)$ & $(-2.70$ to -1.99$)$ \\
\hline Coronary heart disease & -1.33 & -2.58 \\
(ICD-10: I-20-24) & $(-1.49$ to -1.16$)$ & $(-3.90$ to -1.24$)$ \\
Cerebrovascular diseases & -3.27 & -3.14 \\
(ICD-10: I-60-69) & $(-3.57$ to -2.96$)$ & $(-3.50$ to -2.78$)$ \\
Diabetes & 0.67 & -1.92 \\
(ICD-10: E10-15) & $(-0.25$ to 1.59$)$ & $(-2.45$ to -1.39$)$
\end{tabular}

ICD-10 $=$ International Classification of Diseases, $10^{\text {th }}$ revision.

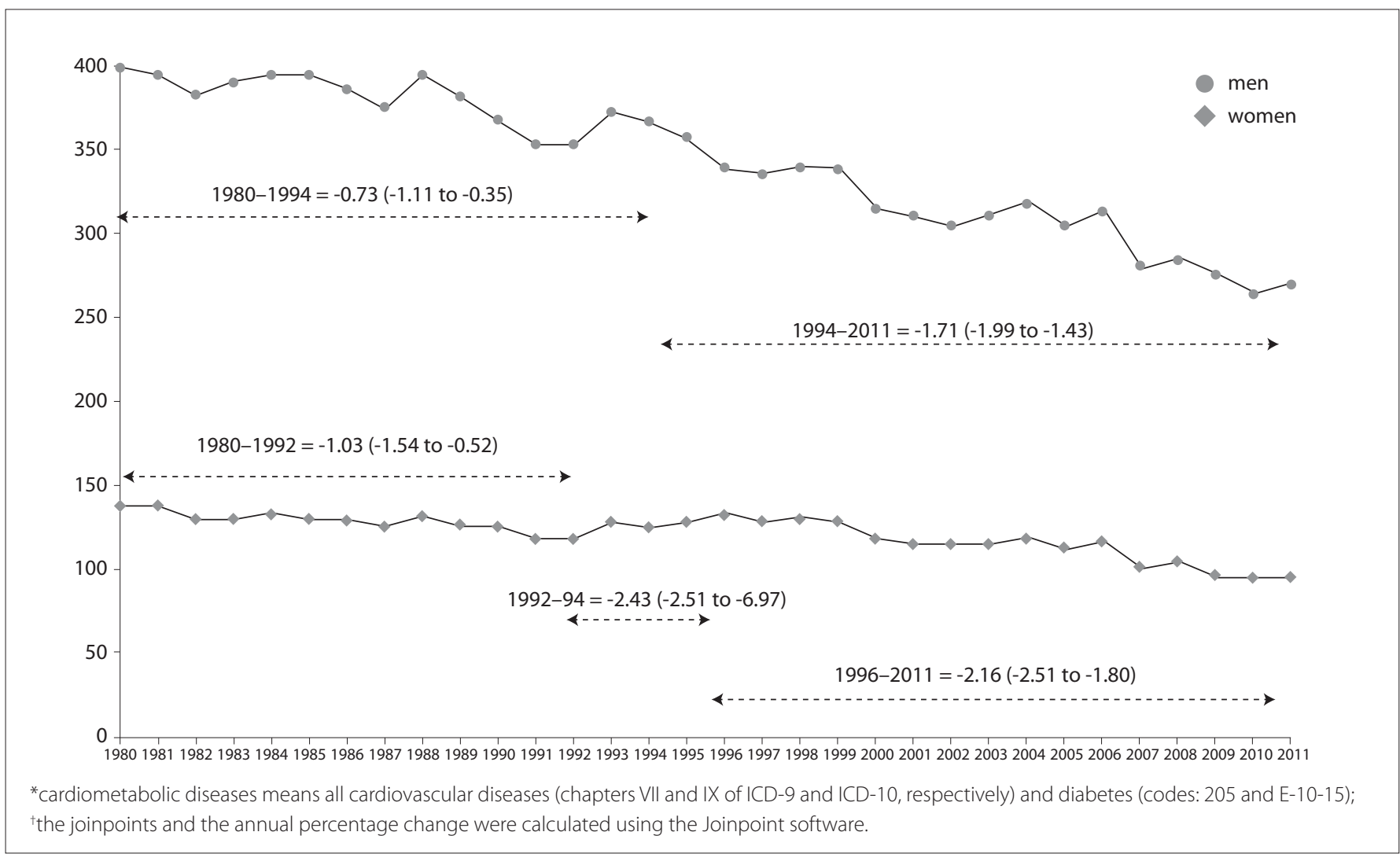

Figure 1. Trends in cardiometabolic disease* death rates in Brazil from 1980 to 2011 with the annual percentage change of each joinpoint ${ }^{\dagger}$ according to sex. 
epidemiologists only in the early 1980s. ${ }^{13}$ Almost all articles on cardiometabolic trends contain classical final remarks such as "further investigation are needed...." Here, this statement is not a cliché. We need to understand the multiple components of this concept called "obesity." Finally, I promise to limit my prophecies over the next three years of my term as editor-chief of this journal.

\section{REFERENCES}

1. Lotufo PA. Increasing obesity in Brazil: predicting a new peak of cardiovascular mortality. Sao Paulo Med J. 2000;1 18(6):161-2.

2. Malta DC, Andrade SC, Claro RM, Bernal RT, Monteiro CA. Trends in prevalence of overweight and obesity in adults in 26 Brazilian state capitals and the Federal District from 2006 to 2012. Rev Bras Epidemiol. 2014;17 Suppl 1:267-76.

3. $\mathrm{Ng} \mathrm{M}$, Fleming $\mathrm{T}$, Robinson $\mathrm{M}$, et al. Global, regional, and national prevalence of overweight and obesity in children and adults during 1980-2013: a systematic analysis for the Global Burden of Disease Study 2013. Lancet. 2014;384(9945):766-81.

4. Lotufo PA, Goulart AC, Fernandes TG, Benseñor IM. A reappraisal of stroke mortality trends in Brazil (1979-2009). Int J Stroke. 2013;8(3):155-63.

5. Moran AE, Forouzanfar MH, Roth GA, et al. Temporal trends in ischemic heart disease mortality in 21 world regions, 1980 to 2010: the Global Burden of Disease 2010 study. Circulation. 2014;129(14):1483-92.

6. van der Leeuw J, van der Graaf Y, Nathoe HM, et al. The separate and combined effects of adiposity and cardiometabolic dysfunction on the risk of recurrent cardiovascular events and mortality in patients with manifest vascular disease. Heart. 2014;100(18):1421-9.

7. Rosamond WD, Chambless LE, Heiss G, et al. Twenty-two-year trends in incidence of myocardial infarction, coronary heart disease mortality, and case fatality in 4 US communities, 1987-2008. Circulation. 2012;125(15):1848-57.

8. Murray CJ, Dias RH, Kulkarni SC, et al. Improving the comparability of diabetes mortality statistics in the U.S. and Mexico. Diabetes Care. 2008;31(3):451-8.

9. Di Cesare M, Bennett JE, Best N, et al. The contributions of risk factor trends to cardiometabolic mortality decline in 26 industrialized countries. Int J Epidemiol. 2013;42(3):838-48.

10. National Cancer Institute. Surveillance Research. Cancer Control and Population Sciences. Statistical Methodology and Applications Branch. Mission Statement. Available from: http://surveillance.cancer. gov/branches/smab/. Accessed in 2014 (Nov 11).

11. Lotufo PA, Benseñor IM. Stroke mortality in Brazil: one example of delayed epidemiological cardiovascular transition. Int J Stroke. 2009;4(1):40-1.

12. Vague J. The degree of masculine differentiation of obesities: a factor determining predisposition to diabetes, atherosclerosis, gout, and uric calculous disease. Am J Clin Nutr. 1956;4(1):20-34.
13. Lapidus L, Bengtsson C, Larsson B, et al. Distribution of adipose tissue and risk of cardiovascular disease and death: a 12 year follow up of participants in the population study of women in Gothenburg, Sweden. Br Med J (Clin Res Ed). 1984;289(6454):1257-61.

\section{Sources of funding: None}

Conflict of interest: None

Date of first submission: October 28, 2014

Last received: December 3, 2014

Accepted: December 3, 2014

\section{Address for correspondence:}

Paulo Andrade Lotufo

Centro de Pesquisa Clínica e Epidemiológica, Hospital Universitário,

Universidade de São Paulo

Av. Prof. Lineu Prestes, 2.565

Butantã — São Paulo (SP) — Brasil

Tel. (+55 11) 3091-9300

E-mail: palotufo@hu.usp.br 